\title{
Ring-Shaped Leiomyoma of the Ciliary Body
}

\author{
Steven H. Tucker $^{\mathrm{a}}$ Eszter Szalai ${ }^{\mathrm{a}}$ Mark Rivellese ${ }^{\mathrm{b}}$ Hans Grossniklaus ${ }^{\mathrm{a}}$ \\ ${ }^{a}$ Department of Ophthalmology, Emory University School of Medicine, and ${ }^{\mathrm{b}}$ Georgia Retina PC, Atlanta, GA, USA
}

\section{Established Facts}

- Uveal mesectodermal leiomyoma is a rare, benign smooth muscle tumor that often presents in women in the second to fourth decade of life.

- The characteristic features used to differentiate leiomyoma from other malignant uveal tumors include the color, transillumination qualities, and ultrasonographic characteristics.

\section{Novel Insights}

- Ciliary body mesectodermal leiomyoma can demonstrate blockage on transillumination with ultrasonographic characteristics of melanoma.

- Ciliary body leiomyoma may present as a ring-shaped tumor and mimic ring-shaped melanoma of the ciliary body.

\section{Keywords}

Leiomyoma $\cdot$ Ciliary body $\cdot$ Melanoma

\begin{abstract}
Purpose: Ciliary body mesectodermal leiomyoma is a rare, benign smooth muscle tumor that typically presents in women in the second to fourth decade of life. The diagnosis is typically based on clinical color, funduscopic appearance, transillumination properties, and B-scan ultrasonography. Methods: We present a patient with a $360^{\circ}$ ciliary body mass with transillumination and ultrasonographic properties consistent with a ring melanoma. Results: This case emphasizes the difficulty in differentiating the benign leiomyoma from similar appearing malignant tumors such as melanoma. Conclusion: The eye was enucleated and the diagnosis was ring-shaped leiomyoma.

(c) 2017 S. Karger AG, Basel
\end{abstract}

\section{KARGER}

(c) 2017 S. Karger AG, Basel

E-Mail karger@karger.com

www.karger.com/oop

\section{Introduction}

Leiomyoma of the ciliary body is a rare and benign tumor that originates from the ciliary body smooth muscle $[1,2]$. It typically exhibits both myogenic and neurogenic characteristics and is considered to be of neural crest origin [3]. Despite its rarity, it has significant clinical ramifications due to both clinical and imaging characteristics which closely resemble other intraocular tumors such as amelanotic melanoma, schwannoma, neurofibroma, lymphoma, and metastatic tumors $[1,2]$. In contrast to leiomyoma, malignant uveal melanoma is the most common primary intraocular tumor found in adults [4]. Both can manifest as a solid vascular mass with a dome shape and smooth surface. Traditionally, leiomyoma transilluminates while melanoma blocks light, but there are anecdotal reports that show blockage due to leiomyoma [2]. 

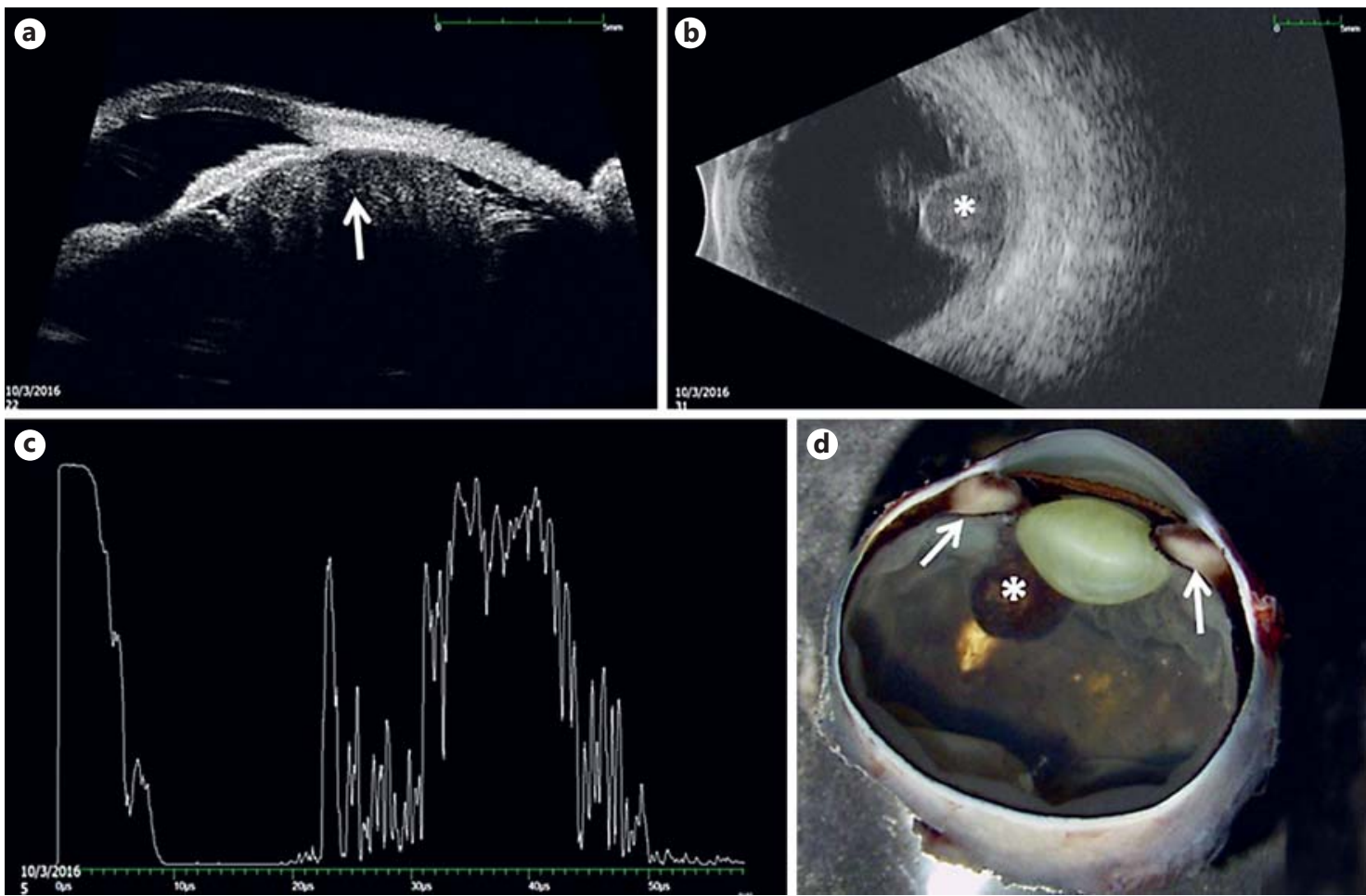

Fig. 1. Ultrasound and gross appearance of the eye. a B scan showing the ciliary body component of the mass (arrow). b B scan also revealing the dome-shaped portion of the mass (asterisk). c A scan showing low internal reflectivity of the mass. $\mathbf{d}$ The enucleated eye contains a circumferential (ring-shaped) tumor in the ciliary body (arrows) as well as a nodular component of the mass (asterisk).

In this report, we describe a large ring-shaped leiomyoma with $360^{\circ}$ infiltration of the ciliary body that had clinical and imaging characteristics consistent with ring-shaped melanoma of the ciliary body.

\section{Case Presentation}

A 36-year-old woman with no significant past ocular history presented with worsening vision in her right eye over the course of 1 year. She noted flashes of light in her right eye and worse vision at night. Her vision was 20/400 and her intraocular pressure was $19 \mathrm{~mm} \mathrm{Hg}$ in the right eye without a relative afferent pupillary defect. The right eye had a mature nuclear sclerotic cataract and demonstrated a large ciliary body cyst with an additional solid component inferonasally.

She was referred for ultrasound biomicroscopy of a ciliary body mass in her right eye, which showed a solid, elevated tumor centered at 5 o'clock with ciliary body involvement. There was low to medium internal reflectivity on A scan with a basal diameter of $17.2 \times 11.5 \mathrm{~mm}$, a thickness of $6.5 \mathrm{~mm}$, and solid echogenicity. These findings were suspicious ultrasonic characteristics for ciliochoroidal melanoma of the right eye (Fig. 1a-c).

Given the presumed diagnosis of a large melanoma based on diagnostic testing, enucleation was performed. The gross pathologic specimen demonstrated blocked transillumination nasally and infe- riorly. There was a white mass in the circumferential ciliary body for $360^{\circ}$ with an inferiorly located $5 \times 5$-mm amelanotic mass (Fig. 1d). Microscopic examination demonstrated an amelanotic mass composed of spindle-shaped cells with bland, vesiculated, fusiform nuclei, and abundant eosinophilic cytoplasm without mitotic figures (Fig. 2a-c). Immunohistochemical stains for smooth muscle actin (SMA) staining were positive (Fig. 2d). Further immunohistochemical stains were negative for estrogen and progesterone receptors. Ultrastructural examination showed a spindle cell tumor with intracytoplasmic 7-9-nm filaments (Fig. 2e, f). A final diagnosis of mesectodermal leiomyoma of the ciliary body was made.

\section{Discussion}

Uveal mesectodermal leiomyoma is a rare, benign tumor first described in the literature in 1950 [5]. In the past 67 years, there has been a limited number of reported cases with no reports of metastases [1]. It characteristically has a predilection for young adult women in their second to fourth decade of life $[2,3]$. Since it is often recognized in young females during reproductive years, there may be a hormonal relationship to the pathogenesis of this tumor [3]. Our patient recognized worsening visual acuity after 

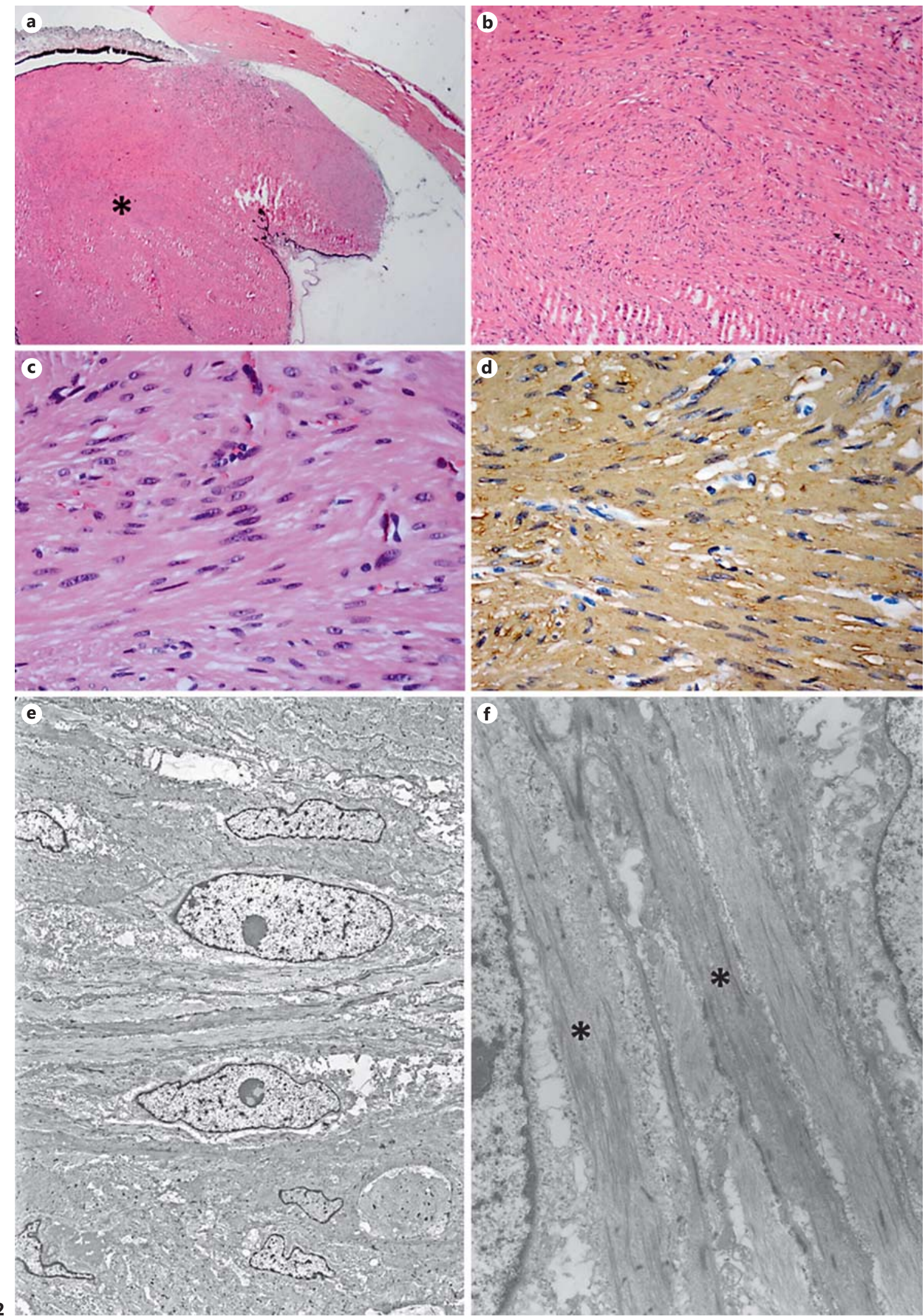

(For legend see next page.) 
pregnancy implicating the role of hormonal changes in the progression of her tumor despite negative immunohistochemical staining. Ciliary body leiomyoma is similar to a uterine fibroid and is often located in the superior ciliary or suprachoroidal space. Clinical features include a yellow-orange color, dome shape, prominent vasculature, and lack of transillumination blockage [2].

Leiomyomas typically demonstrate slow growth in an outward fashion. Ocular manifestations include lens opacification and/or subluxation, glaucoma, and retinal detachment. While benign, the treatment often depends on the size and secondary manifestations of the tumor. Fundus examination is often limited due to lens opacification. If the lesion is clinically visible, it can appear with an orange color or pigmented due to overlying uveal tissue. When possible, uveal leiomyoma will typically show enhanced transillumination compared to blocking which is demonstrated by other malignant tumors [2]. In our case, however, the tumor showed transillumination blockage on the enucleation specimen highlighting the importance of transillumination in the diagnostic process. In situations of limited fundus examination, the diagnosis relies more heavily on imaging.

B-scan ultrasonography often exhibits a dome-shaped mass with increased vascularity, making differentiation from melanoma difficult. MRI imaging has been unable to demonstrate a significant difference between the various intraocular tumors discussed. Biopsy may be useful in establishing a diagnosis. Management of uveal leiomyoma includes observation if it is small and the diagnosis is near certain. For moderate size tumors located at 3-5 o'clock of the iris/ciliary body, the tumor can be removed through partial lamellar sclerouvectomy [2]. Large tumors or unusual cases often require enucleation and/or radiotherapy due to difficulty with diagnosis and sequelae of the large tumor.

Ring-shaped melanomas of the iris and ciliary body are rare entities ( $0.3 \%$ of all uveal melanomas) and the clinical diagnosis is often challenging [6]. Similar to our

Fig. 2. Pathology of the ciliary body tumor. a An eosinophilic tumor is present in the ciliary body. $\mathbf{b}$ The tumor is composed of spindle-shaped cells with abundant cytoplasm. c The spindleshaped cells contain bland nuclei and fibrillar eosinophilic cytoplasm. d Immunohistochemical stain for SMA is positive in the tumor. e Ultrastructural examination showing that tumor cells contain elongated nuclei, some with nucleoli and intracytoplasmic filaments. $\mathbf{f}$ The intracytoplasmic filaments $\left(^{*}\right)$ measure $7-9 \mathrm{~nm}$ in diameter and contain densities. a HE. 5x; b HE. 25×; c HE. 100×. d-f Peroxidase anti-peroxidase. d 100×; e 1,900×; f 7,200×. SMA, smooth muscle actin; HE, hematoxylin and eosin. case, they usually show a circumferential growth pattern and do not cause visual symptoms until advanced stages $[6,7]$. The clinical and imaging characteristics of ring melanomas are often nonspecific, especially in cases of amelanotic ciliary body masses [8]. In our case, the large nonpigmented tumor with $360^{\circ}$ infiltration of the ciliary body had clinical and imaging features consistent with ring-shaped melanoma of the ciliary body. Given these findings, the decision was made to proceed with enucleation. The definitive diagnosis of leiomyoma could only be made with histopathological examination.

\section{Acknowledgment}

Supported in part by NIH P30EY06360 and an unrestricted departmental grant from Research to Prevent Blindness, Inc.

\section{Statement of Ethics}

Case reports on single patients are exempt from IRB approval at our institution. This report is in compliance with HIPAA guidelines.

\section{Disclosure Statement}

The authors have no conflicts of interest to disclose.

References

Ocul Oncol Pathol 2017;3:250-253

Koletsa T, Karayannopoulou G, Dereklis D, Vasileiadis I, Papadimitriou CS, Hytiroglou P: Mesectodermal leiomyoma of the ciliary body: report of a case and review of the literature. Pathol Res Pract 2009;205:125-130.

2 Remmer MH, Kaliki S, Eagle RC, Shields CL, Shields JA: Giant leiomyoma of the ciliary body. Oman J Ophthalmol 2014;7:81-83.

3 Lavric A, Tsimpida M, Hungerford JL, ML Cohen V: Mesectodermal leiomyoma of the ciliary body presenting as anterior staphyloma. Retin Cases Brief Rep 2016, Epub ahead of print.

4 Kaliki S, Shields CL: Uveal melanoma: relatively rare but deadly cancer. Eye (Lond) 2016, Epub ahead of print.

5 Blodi FC: Leiomyoma of the ciliary body. Am J Ophthalmol 1950;33:939-942.

6 Demirci H, Shields CL, Shields JA, Honavar SG, Eagle RC: Ring melanoma of the ciliary body: report on twenty-three patients. Retina 2002;2:698-706.

7 Vásquez LM, Pavlin CJ, McGowan H, Yücel Y, Simpson ER: Ring melanoma of the ciliary body: clinical and ultrasound biomicroscopic characteristics. Can J Ophthalmol 2008;43:229-233.

8 Aziz HA, Modi YS, Plesec TP, Singh AD: Amelanotic irido-ciliary ring melanoma: a clinicopathological correlation. Ocul Oncol Pathol 2016;2:153-155. 